\title{
ARTICLES
}

\section{An introduction to camera trapping of wing-tagged vultures in southern Africa}

\author{
Beryl Wilson
}

McGregor Museum, P.O. Box 316, Kimberley 8300, South Africa

berylwa@gmail.com

http://dx.doi.org/10.4314/vulnew.v69i1.1

\section{Introduction}

Vultures are endangered worldwide, and of South Africa's nine vulture species, seven are already facing various threats of extinction following rapid declines in numbers in recent years (Askew 2014, Ogada et al. 2015).

In East Africa, vultures are primarily threatened by toxic pesticides; whereas in southern Africa they are used for the muthi (traditional medicine) trade, as well as being victims of mass accidental drownings and poisonings, powerline collisions and electrocutions.

Continuous and ongoing monitoring of White-backed Vultures Gyps africanus has taken place on Dronfield Nature Reserve, near Kimberley, Northern Cape, South Africa since 1992. Initially metal and colour-coded leg rings were used but from 2005 onwards, approved plastic patagial (wing) cattle tags were fitted to increase visibility and potential re-sightings or recovery of colour-marked birds. Patagial tags have been used successfully in the study and conservation of many different large raptors across the world (Birds of Prey Working Group 2006, Botha 2007, Martin \& Major 2010).

Several years ago a dedicated Dronfield vulture monitoring site (DVMS) was established on Dronfield Private Nature Reserve, just north of Kimberley, Northern Cape Province, South Africa. Even though it has an observer hide, re- 
sighting records of wing-tagged birds by researchers, farm staff and visitors have been infrequent and anecdotal up until 2011, and it became apparent that an additional method of data collection was needed.

The use of camera traps (also known as wildlife trail cameras) to collect data has become an increasingly popular tool in conservation and ecological research as well as law enforcement. It offers an unobtrusive, unbiased and practical method of supplementing field observations, particularly in remote situations or when personal observation is not possible. They are widely used to monitor rare and elusive species; those that are generally difficult to observe directly, or highly mobile and cryptic species (Carbone et al. 2001). They can also provide detailed knowledge on several ecological components relating to the behavioural ecology of species such as presence/absence data, spatial distribution, relative abundance and population-density estimates, habitat use in space and time, and, if individuals can be identified or marked, population-size estimates and home-range sizes can also be established for some species (Kilshaw \& Macdonald 2011).
With these benefits in mind, a camera trap has been in place at the DVMS since January 2012 that has resulted in significantly increased numbers of re-sighting data. It has also captured important interspecific and intraspecific behavioural interactions, and recorded approximate abundance of target and non-target species at the feeding site.

Since the instrumentation and settings of camera traps were designed with mammals foremost in mind, much of the original trapping success was hampered by the lack of available field techniques for the in situ placement of cameras and their settings appropriate for research of this nature.

This paper aims to highlight the technical aspects regarding camera selection, user settings, field deployment, and data management when used for vulture monitoring in a southern African setting. The advantages and disadvantages of using camera traps for this type of monitoring are also summarised, based on camera trapping experiences over a four-year period.

\section{Use of camera traps in South Africa}

It is important that camera trap users understand their legal responsibilities 
prior to deploying their devices. Currently, the law governing legal surveillance in South Africa is called the Interception and Monitoring Prohibition Act No. 127 of 1992. It is under review, but the legislation is aimed at protecting the privacy of the public and the community. In the case of camera traps used for wildlife monitoring and research purposes, there is no intent to capture images of people, but the onus does fall on the researcher to advise the public and local staff of the presence of the camera traps, although it is not necessary to say where and when the devices will be in use. However, advertising the presence of a camera trap does increase the risk of theft of the device or damage to the equipment. These risks need to be evaluated and appropriate measures taken to prevent or limit losses. Images of people captured during research must be handled appropriately and responsibly, and publication of these images should be avoided.

From an animal ethics viewpoint, no specific current legislation exists in South Africa governing the use of camera trap devices, but researchers can be expected not place the traps in such a way as to harm or injure, or unduly disturb animals in the target area.
Clearly, researchers need permission to deploy the camera trap if they are not themselves the owner or manager of the property on which the device is to be used.

\section{Selection of appropriate equipment}

The camera trap selection process is difficult and needs to take account of budgets, logistics, different climate variables, infrared versus incandescent flashes, physical dimensions of each camera and picture quality. Difficulties also arise because the features and specifications of camera traps vary enormously and this creates a degree of inconsistency between them. However all models have the same basic principle: a photo (and/or video) camera protected by some sort of weather-proof housing, coupled to a mechanism that allows the camera to be triggered automatically when an animal moves in front of it. Virtually all equipment is now digital and these cameras record the photographs (or if the feature is available on the model, video) onto a memory card. Camera traps can be passive (heat in motion sensor) or active (infra-red beam established across a potential path or area). Makes readily available in 
South Africa (in no particular order or preference and not limited to) include: Acorn, Bushnell, Cuddeback, Leupold, Lynx, Minox, Moultrie, Nikko Stirling, Primos, Prostalk, Ranger Outdoor, Reconyx, Rikrhino, Scoutguard, Tasco and Veho. This is a wide variety. Suggested selection criteria include:

\section{Available budget:}

As with most projects, budget restraints often determine the way forward. Aspects to consider from the outset include:

\section{a. Camera costs:}

Currently (2016) digital units range in price from about R1 700 to R19 500 depending on the features available on each unit. Most models that would be ideal for this application will cost about R3 500 currently. Fortunately, monitoring vultures does not require multiple units as with most mammal monitoring surveys.

More importantly, these devices have a limited life and can be expected to last only as long as their warranty period, human and animal tampering aside! They are also generally not repairable and should be considered "throw away" items once they begin to malfunction.

\section{b. Protective housing:}

Most units on the market have optional weather- or tamper-proof housings. They help to disguise and protect the equipment from animals, as well as prevent opportunistic theft or tampering. Expect to pay about R1 500 for the protective box, or consider making a lockable box.

\section{c. Consumables}

Memory cards:

Budget for at least two 16 GB SD cards that can be quickly swapped out. Avoid using $32 \mathrm{~GB}$ cards as you are highly unlikely to utilise them fully and many units cannot accommodate them. Rather spend the extra funds on getting faster (Class 6 or preferably Class 10) 8 or 16 GB cards.

\section{Batteries:}

In this monitoring situation, batteries will need to be changed frequently due to the high number of photographs being recorded in a relatively short time. Most camera traps take four or eight AA batteries. 
Lithium recommended batteries by are manufacturers because of their sustained capacity and high power output and are less affected by extreme cold weather. However a set of eight will cost \pm R220 (2015/2016), but will easily give more than 2000 good quality images depending on the camera settings.

Nickel-Metal Hydride (rechargeable) batteries are initially more expensive but over time will be cost-effective. However, the performance of these batteries differs considerably and they are considerably less reliable.

Alkaline batteries are considerably cheaper, but less dependable in southern African weather conditions and are likely to give less than 400 photos under normal operating conditions.

External battery packs or solar panel connections are available on some units, but concealment of the unit becomes more complicated and could result in increased risks of vandalism and theft, or wildlife tampering.

\section{d. Food provision}

Assuming that monitoring will take place at an already established vulture feeding spot, or water hole, there is still a need to consider the provision of carcasses at the camera site on a regular basis. Since this food is only intended as a supplementary safe feeding lure, carcass provision at the rate of one every 5-10 days is sufficient, but this will depend on how many vultures are present and how quickly the carcasses are completely utilised. If carcasses have to be bought and/or transported to the site there will be additional expenses incurred. Access to storage facilities such as a walk-in freezer will be an advantage when there are surplus carcasses.

\section{Climate variables:}

Consider whether or not the camera can withstand months of harsh climate and wide temperature ranges. Every camera trap on the market is classified as "weather resistant", but some work better in certain conditions. How will the local ambient weather conditions affect the life of the batteries in the camera? Even the Energizer Lithium (R) batteries that boast thousands of photographs are reduced to less than 1000 shots under continuous working conditions in extreme hot and cold temperatures. 
3. Infrared versus incandescent:

This is an old debate but of little concern when camera-trapping vultures. Whilst there is a risk of scaring animals with an incandescent flash, the reward of having a colour night photo versus an infrared camera (which produces only a faint red glow or a black flash in newer and more expensive camera models) in which all the night images are black and white only is obvious. However this not a major concern since vultures are not generally nocturnal, although it is possible to record birds in pre-dawn or postdusk light settings. Vultures have been recorded feeding at night on three occasions at the DVMS.

\section{Detection capabilities:}

Quick trigger speeds (from detection time to the taking of the photo), fast recovery times (time between photos), long detection ranges and wide detection zones are optimal features, especially when camera trapping solitary, fast-moving species like carnivores. The bigger the detection area the camera can monitor and the faster a camera "triggers" and "recovers," the more photos you will have of any given species. Again, these features are somewhat less important when monitoring vultures.

\section{Target species:}

Identifying the species that will be monitored plays the most important part in make and model selection. For use in vulture patagial tag monitoring, aspects like the number of cameras deployed, night vision and detection (trigger) speeds are unimportant. Rather concentrate on a single camera with the highest possible number of megapixels currently available. This will be invaluable when reviewing the images as there is a need to zoom in closely on the recorded images to read the patagial tags. Zooming in on a tag will create blurring of the image and this increases the risk of misinterpreting the alphanumeric codes or having them become indistinguishable at a distance beyond the optimal focus range of the camera trap (Figure 1). 


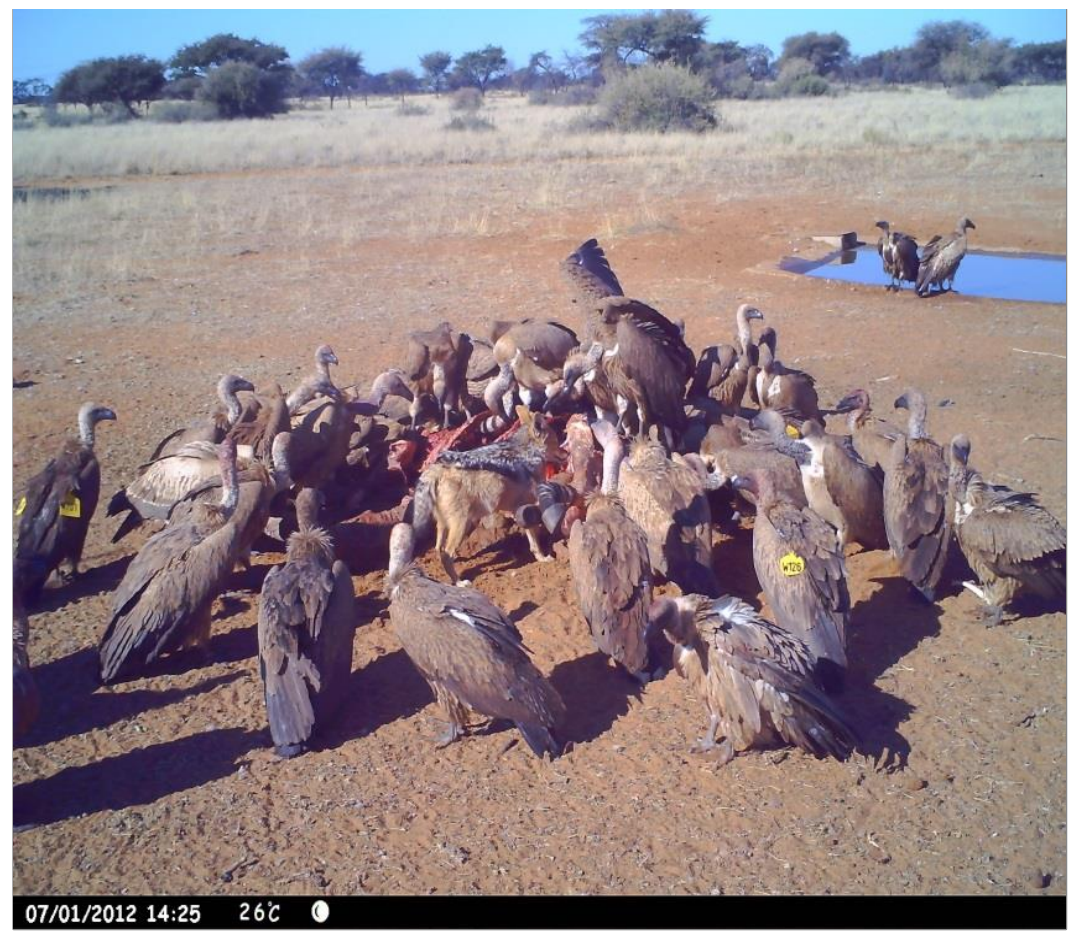

Figure 1: Wing tags are clearly visible, and even when zooming in on tags at a distance they will still be decipherable. Note the additional date, time, temperature and moon phase data captured by the camera trap.

\section{Special features:}

More recently some camera models offer GSM/GPRS features. Operating with a standard SIM card, these cameras can receive SMS commands remotely from a cell phone, as well as being capable of sending images or videos via MMS directly to 1-3 pre-programmed cell phone numbers or email addresses. This makes it possible to have information about current events at the deployment site. This is optional, and will push up initial setup costs as well as ongoing running budgets (for data bundles), but can provide vital information instantly to the researcher in certain situations e.g. the current state of the carcass, activity on site, number of birds present, etc. In the event that the camera trap is stolen, the SIM card can even be locked remotely. 


\section{Camera settings}

The selection of the correct settings from the start means that data will be collected from the outset and disappointments can be avoided. All camera makes and models come with instructions, some more user-friendly than others. Read them anyway! Remember to keep these instructions as you are likely to refer to them again if the original settings are lost, or the camera is deployed in another situation. It is a good idea to download a PDF version of the manual from the manufacturer's website. This can even be loaded onto a cell phone or tablet for field reference purposes.

The final settings that will be used will depend largely on the camera trap itself as well as the research aims, but the following settings are suggested for vulture patagial tag monitoring and should deliver usable data from the first deployment.

1. Time, date stamp, and temperature recording options:

Digital images are stored with time and date data, but for this to be accurate, these values must be set at the time of deployment. Some camera traps will record moon phases, as well as temperature. Note that the temperatures recorded, particularly in daytime temperatures, are not true reflections of ambient temperatures. The recorded temperatures of a camera trap in the shade or one in direct sun will differ dramatically at the same site.

\section{Sensitivity:}

Camera traps are triggered using a passive infrared (PIR) heat-andmotion detector that recognises variation in heat signatures anywhere within the PIR's field of view. How the unit responds to stimuli can be controlled by changing the sensitivity. In high ambient temperatures (above $25^{\circ} \mathrm{C}$ ) the PIR becomes progressively less sensitive to body heat. Conversely, at low ambient temperatures it becomes more sensitive to warmer features. The basic rule of thumb for setting is: situations below $0^{\circ} \mathrm{C}$, select low sensitivity; situations between $0^{\circ} \mathrm{C}$ $25^{\circ} \mathrm{C}$, select normal, and for situations above $25^{\circ} \mathrm{C}$, select high. In this scenario, however, since the camera trap will be aimed downwards to the ground, surface temperatures are usually very high and it is best to select the highest possible setting. However, if for some reason the camera trap is 
required for time-lapse photography, the PIR must be deactivated otherwise the camera will be triggered in between the programmed times.

\section{Trigger speed and delays:}

Trigger speed refers to the time the camera trap takes to "wake up" after movement is detected. This is critical for fast-moving subjects. Camera models with exceptionally high trigger speeds are usually more expensive. For vulture monitoring this is not an important feature as the birds will be spending a considerable time within in the detection range of the camera trap, often in great numbers.

Cameras with a delay period setting or timers can be useful so that the camera only activates during periods of interest. Generally, only daytime images are required since this is when vultures are most likely to be present and feeding. It is recommended that the timer be set to activate the camera trap at least half an hour before sunrise, and deactivate about 45 minutes after sunset. Although other nocturnal information will be lost, you will preserve battery life significantly (no unnecessary photos, especially those requiring the use of the flash) and memory card space.

\section{Number of photos:}

Some camera traps allow for bursts of sequential photos whereas others will only take one photo at a time. Since multiple triggers over an extended time are guaranteed when monitoring vultures, avoid using the photo bursts as this rapidly and unnecessarily depletes the batteries and card storage space. One image per trigger is sufficient. Depending on the size of the memory card, decide whether the older photos will be automatically overwritten or not when the card is full. If cards become full or overwrite themselves on a regular basis, consider using a larger card or downloading more regularly.

\section{Flash setting:}

Flash settings and the various night modes are unimportant for vulture monitoring, but if they are adjustable, make sure that the lowest sensitivity and intensity settings are used, so as to spare the batteries (unless a timer can be used to eliminate night-time shots altogether). 
6. Recovery time:

Closely related to the number of photos, the recovery time of camera traps is important. Recovery time refers to the lag between successive triggers. In other research projects hoping to snap an occasional animal passerby, focus on a quick recovery time is critical and most researchers opt for a more or less continuous burst of images. However, individual vultures will be spending considerable time (up to several hours) in the detection zone, constantly triggering the camera. One (1) minute trigger intervals will result in a large number of repeated images. By reducing the interval period to three (3) minutes between shots, battery life is greatly increased as will the time spent reviewing the downloaded data.

\section{SMS/MMS settings:}

When using a camera trap with these features, take into consideration the objectives of the monitoring. When monitoring elusive predators, it is unlikely that more than a few images are going to be captured per month, and thus setting the unit to send a MMS (usually video) at every trigger event is wise. However, this is usually not the ideal set up for vulture monitoring. Rather, consider setting the camera trap to take an image or 15 second video clip at a predetermined time of day e.g 11:00 and have it delivered to a cell phone and an email address. This will provide a real-time update of the activities of birds (present or absent), state of the carcass, displacement of the camera trap due to tampering, etc. Prior to deployment, remember to disable the SIM pin number, and also to purchase and load data onto the SIM. Also, set a reminder on a calendar to top up this data before it becomes completely depleted to prevent a disruption in the daily service.

\section{Field deployment of a camera trap}

There are a number of general and specific conditions that must be considered when placing a camera trap in the field to optimise the focus area of the images, and to keep the unit in a safe and consistent position.

\section{Access:}

The study area must be accessible to the researcher at all times. Certain areas such as National Parks will not allow researchers unlimited access opportunities, or allow researchers the freedom to move about without 
armed protection if dangerous 2. Camera trap placement:

species are present. Camera traps frequently need routine maintenance that includes cleaning of lenses, angle adjustments, and battery and memory card changes.

One of the most important things to consider when setting up a camera trap is choosing the location in order to get the best results. For vulture monitoring, Figure 2 summarises the recommended positioning features.

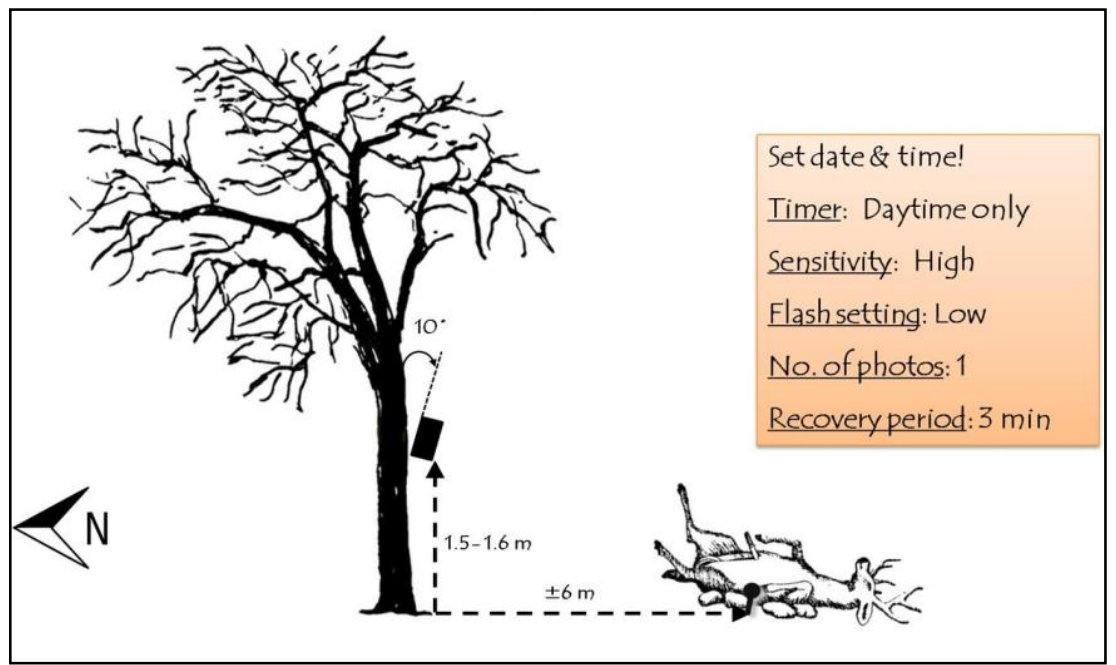

Figure 2: Recommended positioning of a camera trap and lure carcass

\section{a. Height and angle}

Ideally, the camera trap should be placed about $1.5-1.6 \mathrm{~m}$ from the ground and angled $\sim 10^{\circ}$ downwards directly at the carcass. This height above the ground prevents tampering by the vultures (and also other terrestrial animals prone to doing so like hyenas, etc.). Cape Vultures in particular have demonstrated awareness of cameras, and whilst they were not disturbed by the camera's presence, they were 
successful in 'readjusting' it, and on two occasions successfully removed a camera from its attachments to the tree at the DVMS.

\section{b. Direction:}

In the southern hemisphere, orientating the camera traps to the south, south-east or south-west will prevent the likelihood of the camera facing into the sun. This will also help prevent false triggers and improves the image quality.

\section{c. Attachment:}

Camera traps usually come with various mounting devices such as straps with either clips or Velcro fasteners. These are not suitable in this situation as the vultures will bite through the straps if they are accessible. It also makes the unit vulnerable to theft or tampering by monkeys and baboons. If a protective housing is used, it must still be properly mounted. Since all devices differ, each researcher must consider their situation and device, but it is essential to mount the camera trap using at least a metal plate, chain and locks. If a brass lock is used, paint or tarnish the lock to remove the shine that draws unnecessary attention, animal or human, to the camera trap.

\section{d. Anchor device:}

Ideally, a dedicated post at the optimum spot would be the first choice on which to attach the camera trap. However, this is not always an option, and many users are forced to use what is available in the vicinity, normally a tree. For mammal monitoring this would be fine, but with birds this not always ideal. Avoid using a perching tree if possible. Vultures landing and taking off can affect the alignment of the camera trap. If they can reach the camera trap, they will spend hours pecking and tampering with the unit and it will be destroyed. Furthermore, if the camera trap has to be placed below a perching site, the locks need to be protected to avoid corrosion from faecal matter, and the sensor lenses cleaned regularly.

3. Dedicated feeding area:

A very important part of the monitoring success is the permanent placement of a camera trap at a dedicated feeding site such as a vulture restaurant. The specifications of such a site are well-described by 
EWT (2011) but should include the following at least: an open area for flight paths, ample perching opportunities and a shallow water feature suitable for birds to bathe in after feeding.

\section{Food provision:}

Food should be provided regularly and at a rate that the birds can reasonably consume without it becoming their sole food source. All carcasses must be a fresh as possible; veterinary drug-free, and lead-free. Open the abdominal region and deeply score the skin of the entire carcass to allow the birds immediate access to the meat. If possible, turn the carcass over 24 hours later to allow birds to feed on the unexposed sections.

Since the success of the monitoring is directly related to the attracting of birds to the site, any factors that disturb the birds should be avoided. If the site has a viewing hide, signs should be placed requesting visitors not to walk in and around the site, particularly if the birds are present. Panicked birds can easily injure themselves. Over time, resident birds become familiar with a particular person and vehicle. Unfamiliar feeders and drop-off vehicles as well as other disturbances can cause a delay in the arrival of the birds resulting in a deterioration of the carcass. Old carcasses can be turned over to expose the unused side and pulled $15 \mathrm{~m}$ directly away from the camera trap. Often, when visiting a new carcass, some of the birds will find remnant titbits whilst waiting for a feeding opportunity on the fresh carcass, especially if it has rained recently and the hide has rehydrated. Some camera traps will still be able to spot the odd wingtagged bird even at this distance. Permanently ignored food and completely stripped carcasses should be disposed of properly.

It is also highly recommended that a carcass register be kept, either on site if possible or elsewhere, so that the details of the carcass can be recorded. Important information would be: date; species including sex and age; cause of death; provenance / origin of carcass; name of person or organisation that placed the carcass (when carcass provision is from multiple sources or donors).

\section{Food placement:}

Carcasses should be anchored to the ground, particular the smaller species such as warthog, steenbok, impala, springbok, etc. By anchoring the carcass, larger carnivores are 
prevented from dragging the carcass out of the detection zone of the camera, particularly in areas where the feeding site cannot be fenced to exclude them. In addition, the anchoring of carcasses prevents the feeding action of hundreds of vultures from moving the carcass out of range of the camera's PIR and field of view. A large standard, driven into the ground, with a chain and lock is normally sufficient. Vultures will tamper with the lock so ensure a large, high quality, waterproof make is used. Once again, avoiding shiny brass locks which will become a focus of attention for birds.

The field of view for most camera traps is about $43^{\circ}-52^{\circ}$ and carcasses must be centralised in this detection zone. Take the time to use the walk-test function on the unit, or review some test photos in another digital camera or tablet device before permanently positioning either the camera or the carcass anchor spot.

Another advantage of having an anchor is that it marks the ideal position and distance that the carcasses should be placed from the camera, eliminating the guess work each time.

If possible, carcasses should be orientated lengthways away from the camera to maximise side-on viewing opportunities of birds with wingtags, but this is not crucial as the birds move about constantly anyway.
6. Battery and memory card replacement:

Use every food provision opportunity to check the batteries, and exchange the memory card. Take the opportunity to clean the lens and sensor areas. It will quickly become apparent how long a set of batteries lasts in each particular unit. Checking the number of images obtained since the last card switch will also give an indication as to the state of the batteries, as often the battery indicator on the camera trap is inaccurate.

\section{Data management}

Storing camera trap images and processing the data therein requires significant management and effort, so it is best to consider this from the outset rather than attempt a solution at a later point. Much of this will depend on the planned use of the data that can be obtained from the images. Here are some guidance tips: 
1. Data downloading:

It is recommended that on every visit to the camera trap, the memory cards are exchanged rather than just immediately downloading and deleting from the card to make space for the next set of images. An undetected error during the download process can result in the loss of all the images. All memory cards have a limited life-span. These in particular will be constantly hardworking and are going to be subjected to wide temperature fluctuations in the camera trap, and harsh field conditions in general, thus making them more likely to malfunction over time.

\section{Planning:}

Prior to the survey, it is essential to know what the intended use for the data is, as this will largely determine storage and processing procedures. If the sole purpose is to record tagged birds, then it may be acceptable simply to delete the images once they have been assessed. However, most researchers will quickly become aware that each image contains much more information than mere tagged birds and may regret deleting images that they, or other researchers, may have utilised at later point.

\section{Data cleansing:}

Similarly, the final goals of the exercise will determine which images will be kept. As a rule, keep all images - including any apparently irrelevant ones to the project. These may be useful for another purpose. An example from the DVMS was osteophagy (foraging or chewing on bones) by gemsbok at night. After seeing the images, the property manager provided the appropriate mineral licks for the game. It may be desirable, however, to remove all the nocturnal shots of thick-knees feeding on hide beetles and maggots to save some space if someone forgot to the set the timer correctly.

\section{Storing and backups:}

No device is infallible, so the rule is to back-up as much as realistically possible. Download the images from the memory card at the first available opportunity. Use a portable hard drive that can be locked away safely rather than just dumping the images on a PC or laptop, which will quickly become full and progressively slower. When downloading, create a separate folder for each time period 
covered by the images e.g. "201401-30 to 2014-02-18". Using such a format will automatically sort the folders in the correct order and help speed up the retrieval of a specific period or image at a later point.

Do not work directly from the memory cards when reviewing the images as this is additional wearand-tear. Most camera trap manufacturers recommend not formatting the cards in a computer but to rather use the built-in camera memory feature to do so. Do not use these memory cards in other devices unless they are re-formatted again when replaced back in the camera trap.

\section{Data sheet design:}

This will depend on the end goal, but a basic Excel file will suffice. Consider creating several sheets in one file because this allows calculations to be performed. For example, Sheet 1 can contain the following: date; time of arrival of first vulture; time of departure of the last vulture; maximum number of each species of vultures recorded in the field of view; number of crows present; other raptors present; wingtag numbers / colour rings etc. Sheet 2 could then contain running "counts" of the number of times a particular individual has been recorded as present (on Sheet 1), and also keeps complete running totals of tagged birds. For the purposes of data collection on this project, a resighting record was considered as a bird being present at least once on that day, regardless of how many times it appears in images. If the bird is present the following day, this is a new re-sighting record.

\section{Reviewing images:}

After much trial-and-error, and in the absence of using a dual-screen computer, it is strongly recommended that images be downloaded to a tablet device whilst the re-sighting data are added to PC or laptop. Tablet devices have simple, swift zoom features and excellent pixel quality allowing even birds at a fair distance from the focal centre of the camera to still be quickly viewed. The switching back and forth between a viewing programme and the data sheet on a single-screened computer is cumbersome and tiring, and typo errors are more likely to occur.

Amongst the thousands of downloaded images, there are always several interesting ones that may be needed for presentations or reports. Copy these immediately to a 
dedicated folder for quick access later. It is very frustrating trying to locate a single, prized image after several months on a hard drive with 30000 images on it.

\section{Analysing data:}

This completely depends on the nature of the project, or it may be just basic monitoring. There is a wealth of data that can be retrieved from images, even at a much later date. This includes information about ambient temperatures and moon phases; presence/absence; population trends; animal activity and abundance; individual time spent at a carcass; intra- and interspecific behaviour; carcass utilisation, and even individual health (e.g. the monitoring of birds with broken legs etc.).

8. Data sharing:

As the wing-tagging programme is being conducted throughout southern Africa, it is essential to share re- sightings data, especially since the vultures are so wide-ranging. Apart from submitting re-sightings data to the Birds of Prey Programme, it is courteous and sensible to inform other regions as to the presence of one of their birds in your region. The favour is sure to be returned at some point!

\section{Acknowledgements}

I would like to express grateful thanks to all the researchers and volunteers across southern Africa who have annually wing-tagged birds under the guidance of the Endangered Wildlife Trust's Birds of Prey Programme. The DVMS is grateful for the sponsorship of two camera traps and three years' worth of batteries by the Hawk Conservancy Trust, UK and a single a camera trap from Gauntlet Birds of Prey, UK. Carcasses are regularly donated by Wintershoek Safaris, De Beers Consolidated Mines, AIS Farming, Marrick Safaris, and the local Stock Theft Unit of SAPS. 
Summary of the advantages and disadvantages of using a camera trap for vulture monitoring

\begin{tabular}{|c|c|}
\hline Advantages & Disadvantages \\
\hline 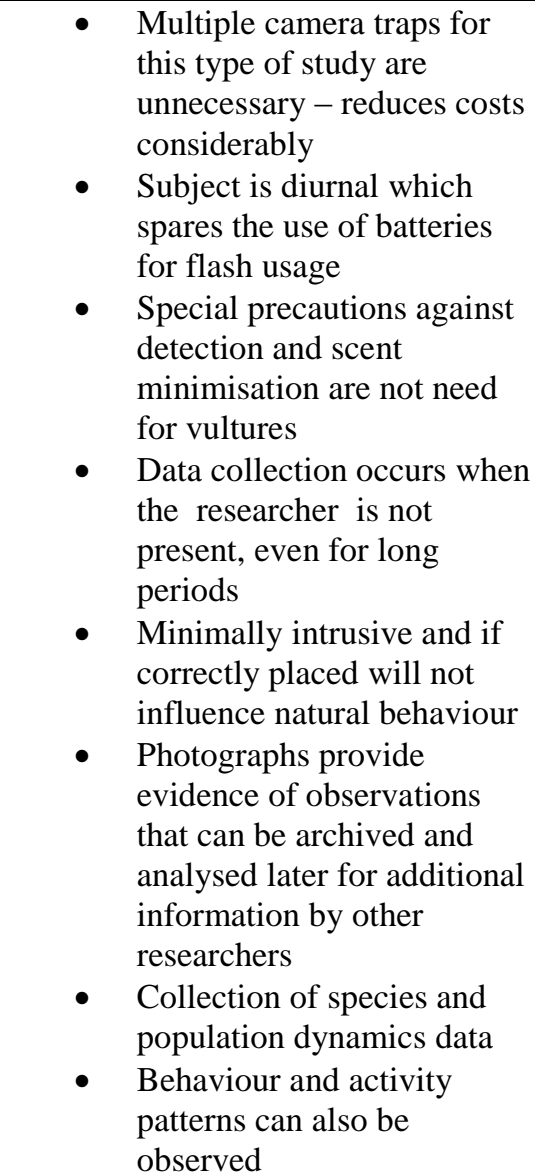 & $\begin{array}{l}\text { - Initial capital investment can } \\
\text { be costly, plus allowance for } \\
\text { batteries (and cellular data if } \\
\text { used) need to be budgeted } \\
\text { Time consuming analysing } \\
\text { the data } \\
\text { - Cameras collect restricted } \\
\text { data owing to the small field } \\
\text { of view area } \\
\text { Cameras are vulnerable to } \\
\text { human and animal } \\
\text { vandalism } \\
\text { Procurement of regular food } \\
\text { supplies for regular baiting } \\
\text { can be expensive or } \\
\text { problematic } \\
\text { Ideally, a dedicated water } \\
\text { point and feeding area needs } \\
\text { to be established } \\
\text { Devices have a limited life } \\
\text { in the South African climate } \\
\text { - usually the length of their } \\
\text { warranty } \\
\text { Devices are generally not } \\
\text { repairable and are best } \\
\text { considered "throw-away" } \\
\text { items }\end{array}$ \\
\hline
\end{tabular}


Keywords: camera traps, trail cameras, vultures, monitoring

\section{References}

Ancrenaz, M., Hearn, A.J., Ross, J., Sollmann, R. \& Wilting, A. 2012. Handbook for wildlife monitoring using camera-taps. BBEC II Secretariat, Malaysia.

Askew, N. 2014. Vultures in Africa and Europe could face extinction within our lifetime warn conservationists. www.birdlife.org/worldwide/news/vultures-africa-and-europe-could-faceextinction-within-our-lifetime-warn

Birds of Prey Working Group. 2006. Policy and protocol for the colourmarking of southern African vultures. Sasol \& Endangered Wildlife Trust, Birds of Prey Working Group, Johannesburg, South Africa.

Botha, A. 2007. A review of colour-marking techniques used on vultures in southern Africa. Vulture News 56: 52-63.

Bury, R. Mammal Surveying - Wildlife Camera Trapping and Environmental photography in the Highlands of Scotland. www.ronburyswildlife.com

Carbone, C., Coulson, T., Christie, S., Conforti, K., Seidensticker, J., Franklin, N., Tilson, R., Ginsberg, J. R., Kinnaird, M., Laidlaw, R., Lynam, A., O'Brien, T., Wan Shahruddin, W. N., Griffiths, M., Holden, J., Martyr, D., Kawanishi, K., Sunquist, M., Macdonald, D. W., Nath, L., McDougal, C. \& Smith, D. J. L. 2001. The use of photographic rates to estimate densities of tigers and other cryptic mammals. Animal Conservation 4: 75-79. 
Endangered Wildlife Trust. 2011. The Sasol Guide to the Establishment and Operation of Supplementary Feeding Sites for Vultures. Birds of Prey Programme.

http://www.ewt.org.za/toolkits/booklets/Vulture\%20Restuarant\%20bookle t.pdf

Kilshaw, K. \& Macdonald, D.W. 2011. The use of camera trapping as a method to survey for the Scottish wildcat. Scottish Natural Heritage Commissioned Report No. 479.

Martin, J.M. \& Major, R.E. 2010. The use of cattle ear-tags as patagial markers for large birds - a field assessment on adult and nestling Australian White Ibis. Waterbirds 33: 264-268.

Meek, P., Ballard, G. \& Fleming, P. 2012. An introduction to camera trapping for wildlife surveys in Australia. PestSmart Toolkit publication, Invasive Animals Cooperative Research Centre, Canberra, Australia.

Ogada, D., Shaw, P., Beyers, R.L., Buij, R., Murn, C., Thiollay, J.M., Beale, C.M., Holdo, R.M., Pomeroy, D., Baker, N., Krüger, S.C., Botha, A., Virani, M.Z., Monadjem, A. \& Sinclair, A.R.E. 2015. Another continental vulture crisis: Africa's vultures collapsing toward extinction. Conservation Letters DOI - 10.1111/conl.12182.

Ryan, P.G., Shaw, J.M., Van der Merwe R. \& Van der Merwe, E. 2012. Carrion attraction: Goshawks and other birds captured on camera trap. Ornithological Observations 3: 102-106.

$* * * * * *$ 